\title{
Estudo de pacientes com a forma surto-remissão cadastrados na Secretaria de Estado da Saúde de Minas Gerais
}

\author{
Eustáquio Claret dos Santos ${ }^{1,2,3}$, MiticoYokota ${ }^{1}$, Nádia Fernanda Rezende Dias ${ }^{3}$
}

\begin{abstract}
RESUMO - Esclerose múltipla é uma doença crônica, inflamatória e desmielinizante do sistema nervoso central. O tratamento preconizado para a forma surto-remissão é com imunomoduladores, fornecidos em Belo Horizonte pela Secretaria de Estado da Saúde de Minas Gerais (SES/MG). Analisamos os dados de 283 pacientes inscritos no Programa de Medicamentos Excepcionais (imunomoduladores) da SES/MG no período de maio a outubro de 2004 . Houve predomínio do sexo feminino $(3,7: 1)$ e a idade média dos pacientes foi $38,4 \pm 10,7$ anos, com as primeiras manifestações clínicas ocorridas aos $29,7 \pm 10,2$ anos. Os sintomas iniciais foram: sensitivos $(46,4 \%)$, visuais $(33,1 \%)$, síndrome de tronco cerebral/cerebelo $(30,1 \%)$ e motores $(25,9 \%)$. Os sintomas observados em surtos subseqüentes foram motores $(22,3 \%)$, síndromes de tronco cerebral/cerebelo (18,9\%), visuais (18,9\%) e sensitivos (9,6\%). Em 234 RM de encéfalo e 109 RM da medula espinhal existiam lesões desmielinizantes em $97,4 \%$ e $85,3 \%$ respectivamente. Comparamos nossos achados com outras séries descritas na literatura brasileira.
\end{abstract}

PALAVRAS-CHAVE: esclerose múltipla, forma surto-remissão, doenças desmielinizantes.

\section{Multiple sclerosis: study of patients with relapsing-remitting form registered at Minas Gerais Secretary State for Health}

\begin{abstract}
Multiple sclerosis is a chronic inflammatory demyelinating disease of the central nervous system. The recommended treatment for the relapsing-remitting form is carried out with the immunomodulatory drugs, which are provided, in Belo Horizonte, by Minas Gerais Secretary of State for Health (MG/ $\mathrm{SSH}$ ). We analyzed data of 283 patients registered at Exceptional Drug Program (immunomodulators) of SES/MG from May to October 2004. There was predominance of females (3.7:1) and the mean age was $38.4 \pm 10.7$ years old. The first clinical manifestations were $29.7 \pm 10.2$ years old. The initial symptoms included sensorial (46.4\%), visual (33.1\%), brainstem/cerebellar syndromes (30.1\%) and motor (pyramidal) syndrome $(25.9 \%)$. The symptoms observed in subsequent relapses were motor (22.3\%), brainstem/cerebellar syndromes (18.9\%), visual (18.9\%) and sensorial findings (9.6\%). In 234 brain MRI and 109 spine MRI there were demyelinating lesions at $97.4 \%$ and $85.3 \%$ respectively. We compared our findings with other series described on Brazilian literature.
\end{abstract}

KEY WORDS: multiple sclerosis, relapsing-remitting form, demyelinating diseases.

A esclerose múltipla (EM) é uma doença crônica, inflamatória, auto-imune e desmielinizante do sistema nervoso central (SNC). Acomete pacientes jovens, principalmente na faixa etária dos 20 aos 40 anos, que corresponde à fase de maior produtividade dos indivíduos. Provoca incapacidades funcionais progressivas, causando grande impacto na vida dos portadores e de seus familiares. A principal forma de evolução da doença é a surto-remissão (EMSR), para a qual se preconiza atualmente tratamento medicamentoso com imunomoduladores ${ }^{1}$. Há também outras apresentações clínicas, como primariamente progressiva, secundariamente progressiva e progressiva com surtos ${ }^{2}$.

Os imunomoduladores (interferon beta- $1 \mathrm{a}, 1 \mathrm{~b}$ e acetato de glatiramer) reduzem a freqüência e a gravidade dos surtos, alterando assim a progressão da doença, quando na forma surto-remissão $0^{3,4}$. Estes medicamentos têm sido supridos pelo Poder Públi-

${ }^{1}$ Secretaria de Estado da Saúde de Minas Gerais / Diretoria de Redes Assistenciais / Coordenadoria de Alta Complexidade; ${ }^{2}$ Faculdade de Ciências da Saúde - Universidade FUMEC, Belo Horizonte MG, Brasil; ${ }^{3}$ Serviço de Neurologia e Neurofisiologia Clínica do Hospital Socor, Belo Horizonte MG, Brasil.

Recebido 22 Janeiro 2007, recebido na forma final 18 Maio 2007. Aceito 30 Junho 2007. 
Tabela 1. Faixa etária dos pacientes portadores de EMSR de Belo Horizonte cadastrados na SESIMG.

\begin{tabular}{|c|c|c|c|c|c|}
\hline \multirow{2}{*}{$\begin{array}{c}\text { Faixa etária } \\
\text { (anos) }\end{array}$} & \multicolumn{2}{|c|}{ Masculino } & \multicolumn{2}{|c|}{ Feminino } & \multirow[t]{2}{*}{ Tota } \\
\hline & $\mathrm{n}$ & $\%$ & $\mathrm{n}$ & $\%$ & \\
\hline 11 a 20 & 0 & 0,0 & 8 & 3,6 & 8 \\
\hline 21 a 30 & 18 & 30,0 & 52 & 23,3 & 70 \\
\hline 31 a 40 & 18 & 30,0 & 60 & 26,9 & 78 \\
\hline 41 a 50 & 15 & 25,0 & 72 & 32,2 & 87 \\
\hline 51 a 60 & 5 & 8,3 & 29 & 13,1 & 34 \\
\hline 61 a 70 & 4 & 6,7 & 2 & 0,9 & 6 \\
\hline Total & 60 & 100 & 223 & 100 & 283 \\
\hline
\end{tabular}

co, como previsto na Lei Federal 8080, de 19.09.1990, que dispõe sobre o Sistema Único de Saúde, com destaque para o inciso $\mathrm{VI}$, do art.6, que regulamenta o direito de acesso a medicamentos para tratamento de saúde. Em 2001 o Ministério da Saúde desenvolveu o Protocolo Clínico e Diretrizes Terapêuticas para dispensação de imunomoduladores na EM, nos termos da Portaria ${ }^{\circ} 97$ da Secretaria de Assistência à Saúde ${ }^{5}$. No Estado de Minas Gerais estes medicamentos são disponibilizados pela Coordenadoria de Medicamentos Excepcionais da Superintendência de Atenção à Saúde da Secretaria de Estado da Saúde de Minas Gerais (SES/MG).

Com o objetivo de contribuir para o estudo epidemiológico da EM no Brasil, descrevemos as características dos pacientes portadores de EMSR cadastrados no programa de dispensação de medicamentos excepcionais de Belo Horizonte e beneficiados pelo recebimento dos imunomoduladores.

\section{MÉTODO}

Estudo descritivo de 283 casos de EMSR, do município de Belo Horizonte, MG, Brasil, realizado no período de maio a outubro de 2004. Os dados foram obtidos a partir da análise de questionários médicos constantes nos Processos de Solicitação de Medicamentos Excepcionais da SES/ MG. Estes processos foram deferidos pela Coordenadoria de Esclerose Múltipla do Estado de Minas Gerais, unidade administrativa da SES/MG, de acordo com os critérios de inclusão e exclusão de pacientes do Protocolo Clínico e Diretrizes Terapêuticas - EMSR, da Portaria SAS n $97^{5}$. Após deferimento os processos foram autuados, protocolados e são renovados trimestralmente no citado órgão estadual. Os principais critérios de inclusão e exclusão de pacientes obedecem à Portaria SAS/MS nº 97 de 22 de Março de $2001^{5}$.

Consideramos os relatos de sexo, idade, manifestações clínicas, além da revisão dos resultados de RM de encéfalo (234) e de medula espinhal (109), líquido cefalorraquidiano (LCR) (128) e potenciais evocados (126), que colaboraram no diagnóstico, segundo o Painel Internacional para o Diagnóstico da EM, publicado em 2001 e revisado em $2005^{6-8}$. Os dados levantados foram organizados no programa EPI-INFO (versão 6.01) e analisados estatisticamente.
Tabela 2. Localização das lesões encefálicas sugestivas de doença desmielinizante à RM do encéfalo em 234 portadores de EMSR.

\begin{tabular}{lcc}
\hline Localização & $\mathrm{N}^{\circ}$ de pacientes & $\%$ \\
\hline Periventricular & 176 & $75,2 \%$ \\
Corpo caloso & 137 & $58,5 \%$ \\
Subcortical & 114 & $48,7 \%$ \\
Infratentorial & 114 & $48,7 \%$ \\
Centro semi-oval & 61 & $26,1 \%$ \\
Nervo óptico & 22 & $9,4 \%$ \\
Tálamo & 15 & $6,4 \%$ \\
Núcleos da base & 9 & $3,9 \%$ \\
\hline
\end{tabular}

Os pacientes assinaram termo de consentimento pós-informado. O estudo foi submetido e aprovado pelo Comitê de Ética em Pesquisa do Hospital SOCOR - Belo Horizonte.

\section{RESULTADOS}

A idade média dos pacientes foi $38,4 \pm 10,7$ anos e a Tabela 1 ilustra a relação entre a faixa etária estratificada e o sexo dos pacientes. A proporção feminino:masculino foi 3,7:1. A idade média das primeiras

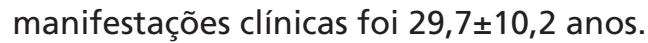

Os sintomas iniciais mais comuns foram: sensitivos $(46,4 \%)$, visuais $(33,1 \%)$, síndromes de tronco cerebral/cerebelo $(30,1 \%)$ e motores $(25,9 \%)$. Os sintomas evolutivos se caracterizaram da seguinte maneira: motores $(22,3 \%)$, síndromes de tronco cerebral/cerebelo $(18,9 \%)$, visuais $(18 \%)$ e sensitivos $(9,6 \%)$.

Em 234 RM de encéfalo havia lesões desmielinizantes compatíveis com o diagnóstico de EM em 228 $(97,4 \%)$. As localizações das lesões são descritas na Tabela 2. Deve-se considerar que cada paciente pode apresentar lesões em mais de uma região encefálica. A RM da medula espinhal foi realizada em 109 pacientes e 93 deles $(85,3 \%)$ apresentaram alterações compatíveis com EM.

Os exames de LCR de 128 pacientes tiveram o seguinte resultado: normal (44,5\%), IgG aumentada e ausência de bandas oligoclonais (34,4\%), IgG normal 
Tabela 3. Resultados de estudos epidemiológicos sobre esclerose múltipla no Brasil.

\begin{tabular}{|c|c|c|c|c|c|c|}
\hline Autores & Amostra & $\mathrm{F}: \mathrm{M}$ & Forma clínica & Idade média & Sintoma & ais (\%) \\
\hline $\begin{array}{l}\text { Callegaro }^{9} \\
(1989)\end{array}$ & 120 & $1,6: 1$ & $\begin{array}{c}85 \% \text { SR } \\
10,2 \% \text { PP } \\
4,2 \% \text { SP }\end{array}$ & $27,9 \pm 8,9$ & $\begin{array}{c}\mathrm{M}=45 \\
\mathrm{TC} / \mathrm{C}=30,7\end{array}$ & $\begin{array}{l}S=30,8 \\
V=14,1\end{array}$ \\
\hline $\begin{array}{l}\text { Leite et al. }{ }^{10} \\
(1990)\end{array}$ & 51 & $2,1: 1$ & $\begin{array}{c}47 \% \text { SR } \\
19,7 \% \text { PP } \\
33,3 \% \text { mista }\end{array}$ & $34,5 \pm 13,9$ & $\begin{array}{l}\mathrm{M}=54,1 \\
\mathrm{TC} / \mathrm{C}=3\end{array}$ & $\begin{array}{l}S=41,6 \\
V=35,4\end{array}$ \\
\hline $\begin{array}{l}\text { Lana-Peixoto }^{11} \\
(1992)\end{array}$ & 67 & $2,3: 1$ & - & $28,9 \pm 10,4$ & $\begin{array}{c}\mathrm{M}=51 \\
\mathrm{TC} / \mathrm{C}=34\end{array}$ & $\begin{array}{l}\mathrm{S}=39 \\
\mathrm{~V}=43\end{array}$ \\
\hline $\begin{array}{l}\text { Tilbery et al. }{ }^{12} \\
\text { (1995) }\end{array}$ & 214 & 2,9:1 & $\begin{array}{l}82 \% \text { SR } \\
18 \% \text { PP }\end{array}$ & 28,0 & $\begin{array}{c}M=47 \\
\mathrm{TC} / \mathrm{C}=32\end{array}$ & $\begin{array}{l}S=27 \\
V=27\end{array}$ \\
\hline $\begin{array}{l}\text { Papaiz-Alvarenga et al. }{ }^{13} \\
\text { (1995) }\end{array}$ & 88 & $3: 1$ & $\begin{array}{c}88,6 \% \text { SR } \\
4,5 \% \text { PP } \\
6,8 \% \text { SP }\end{array}$ & $27,9 \pm 11,3$ & $\begin{array}{c}\mathrm{M}=52 \\
\mathrm{TC} / \mathrm{C}=50\end{array}$ & $\begin{array}{l}S=51 \\
V=29\end{array}$ \\
\hline $\begin{array}{l}\text { Oliveira et al. }{ }^{14} \\
\text { (1999) }\end{array}$ & 50 & $2: 1$ & $\begin{array}{l}60 \% \text { SR } \\
30 \% \text { PP } \\
10 \% \text { SP }\end{array}$ & $32,5 \pm 9,6$ & $\begin{array}{c}\mathrm{M}=70 \\
\mathrm{TC} / \mathrm{C}=48\end{array}$ & $\begin{array}{l}S=16 \\
V=10\end{array}$ \\
\hline $\begin{array}{l}\text { Moreira et al. }{ }^{15} \\
(2000)\end{array}$ & 302 & $\begin{array}{l}3,5: 1 \\
(S R)\end{array}$ & $\begin{array}{c}72,8 \% \text { SR } \\
13,6 \% \text { PP } \\
13,6 \% \text { SP } \\
19,8 \% \text { benigna }\end{array}$ & $28,7(S R)$ & $\begin{array}{c}\mathrm{M}=28,5 \\
\mathrm{TC} / \mathrm{C}=15,8\end{array}$ & $\begin{array}{l}S=29,5 \\
V=23,5\end{array}$ \\
\hline Arruda et al. ${ }^{16}$ (2001) & 200 & $1,8: 1$ & $\begin{array}{c}91 \% \mathrm{SR} \\
8 \% \mathrm{PP} \\
1 \% \mathrm{SP}\end{array}$ & $32 \pm 9,9$ & $\begin{array}{l}\mathrm{M}=49,5 \\
\mathrm{TC} / \mathrm{C}=63\end{array}$ & $\begin{array}{c}S=53 \\
V=39,5\end{array}$ \\
\hline $\begin{array}{l}\text { Brito Ferreira et al. }{ }^{17} \\
(2004)\end{array}$ & 118 & $\begin{array}{l}4,1: 1 \\
(S R)\end{array}$ & $\begin{array}{c}70,4 \% \text { SR } \\
5,9 \% \text { PP } \\
23,7 \% \text { SP }\end{array}$ & $33,2 \pm 11,5$ & - & - \\
\hline $\begin{array}{l}\text { Fragoso, Fiore }{ }^{18} \\
(2005)\end{array}$ & 81 & $3,3: 1$ & $82,7 \%$ SR & 32 & - & - \\
\hline $\begin{array}{l}\text { Cardoso et al. }{ }^{19} \\
(2006)\end{array}$ & 121 & $4: 1$ & $\begin{array}{c}91,3 \% \text { SR } \\
6,1 \% \text { PP } \\
1,7 \mathrm{SP}\end{array}$ & $31.1 \pm 11.02$ & $\begin{array}{c}\mathrm{TC} / \mathrm{C}=11,8 \\
\mathrm{~V}=34,4\end{array}$ & \\
\hline $\begin{array}{l}\text { Grzesiuk }^{20} \\
(2006)\end{array}$ & 20 & $3: 1$ & $\begin{array}{c}75 \% \text { SR } \\
5 \% \text { PP } \\
20 \% \text { SP }\end{array}$ & 33,8 & $\begin{array}{c}\mathrm{M}=90 \\
\mathrm{TC} / \mathrm{C}=60\end{array}$ & $V=25$ \\
\hline $\begin{array}{l}\text { Presente série } \\
\text { (2006) }\end{array}$ & $\begin{array}{l}283 \\
(\mathrm{SR})\end{array}$ & $\begin{array}{c}3,7: 1 \\
(S R)\end{array}$ & $100 \% \mathrm{SR}$ & $29,7 \pm 10,2$ & $\begin{array}{c}\mathrm{M}=25,9 \\
\mathrm{TC} / \mathrm{C}=30,1\end{array}$ & $\begin{array}{l}S=46,4 \\
V=33,1\end{array}$ \\
\hline
\end{tabular}

SR, surto remissão; PP, primariamente progressiva; SP, secundariamente progressiva; $M$, alterações motoras; $S$, alterações sensitivas; TC/C, alterações tronco cerebral/cerebelo, $\mathrm{V}$, alterações visuais.

e bandas oligoclonais presentes $(11,7 \%)$, IgG aumentada e bandas oligoclonais presentes $(9,4 \%)$.

Foram realizados 66 potenciais evocados visuais, dos quais $83,6 \%$ estavam alterados. O potencial evocado auditivo de tronco cerebral foi realizado em 24 pacientes, sendo que $50 \%$ apresentavam resultados alterados e 36 pacientes fizeram o potencial evocado somatossensitivo, com anormalidades em $72,2 \%$. Comparamos nossos dados com os resultados de várias casuísticas realizadas no Brasil, nos anos de 1989 até 2006, nos Estados de Minas Gerais, São Paulo, Rio de Janeiro, Paraná, Pernambuco, Bahia e Mato Grosso, como mostra a Tabela $3^{9-20}$.

\section{DISCUSSÃO}

A maioria dos resultados dos estudos se refere a todas as formas evolutivas de EM e não exclusivamente à forma surto-remissão, característica de nossa série. A maior freqüência de EM no sexo feminino $(3,7: 1)$ foi relatada por todos os autores das outras séries brasileiras, com variações entre 1,6:19 $\mathrm{e}$ $4,1: 1^{17}$. A média de idade do início dos sintomas em nosso estudo foi $29,7 \pm 10,2$ anos, assemelhando-se às outras casuísticas nacionais, que foram de $27,9^{9,13}$ a $34,5 \operatorname{anos}^{10}$. Com relação às manifestações clínicas, a variação observada entre o nosso estudo e os demais 
pode ser explicada, em parte, pela análise realizada por alguns autores de diferentes formas evolutivas da doença e não apenas da forma surto-remissão.

As alterações encontradas nas RM se aproximam daquelas observadas em um estudo na cidade de Curitiba, de 107 casos $^{21}$. Nesta série as regiões encefálicas mais acometidas foram: periventricular (82,7\%), centro semi-oval (48\%), corpo caloso/região pericalosa $(37,8 \%)$ e estruturas infratentorias como ponte $(28,6 \%)$, hemisfério cerebelar $(14,3 \%)$ e pedúnculo cerebelar (13,3\%).

Os resultados dos exames de LCR não mostraram, a princípio, relação com a EMSR. Devemos considerar que não houve padrão nos dados do LCR, pois há diferenças no estágio clínico em que se fez a coleta, os exames foram realizados em vários laboratórios diferentes e também faltam dados, em alguns processos, a respeito da contagem de células e avaliação quantitativa de IgG. Vale ressaltar que em um estudo de 86 pacientes com EM forma clinicamente defini$\mathrm{da}$, os dados obtidos sugeriram que as características clínicas não parecem ter influência na contagem de células e na presença de bandas oligoclonais ${ }^{22}$.

A realização dos potenciais evocados cumpre a função de colaborar no diagnóstico, buscando lesões subclínicas do SNC nos casos suspeitos de EM e se restringe, atualmente, ao estudo do potencial evocado visual ${ }^{8}$.

Finalmente, vale ressaltar que os dados aqui apresentados são apenas uma pequena amostra de uma doença que a todo instante nos desafia. A criação de Centros de Referência em esclerose múltipla, operantes, além das atividades assistenciais, poderão ser um importante instrumento de ensino e pesquisa e permitirão um conhecimento mais amplo desta doença em nosso meio.

\section{REFERÊNCIAS}

1. Noseworthy JH, Lucchinetti C, Rodriguez M, Weinshenker BG. Multiple sclerosis. N Engl J Med 2000;343:938-951.

2. Lublin FD, Reingold SC. Defining the clinical course of multiple scle- rosis: results of an international survey. National Multiple Sclerosis Society (USA) Advisory Committee on Clinical trials of New Agents in Multiple Sclerosis.Neurology 1996;46:907-911.

3. Tilbery CB, Moreira MA, Mendes MF, Lana-Peixoto MA. and The Brazilian Committee for Treatment and Research in Multiple Sclerosis, BCTRIMS. Recomendações para o uso de drogas imunomoduladoras na esclerose múltipla. Arq Neuropsiquiatr 2000;58:769-776.

4. Lana-Peixoto MA, Callegaro D, Moreira MA, et al. Consenso expandido do BCTRIMS para o tratamento da esclerose múltipla. III: diretrizes baseadas em evidências e recomendações. Arq Neuropsiquiatr 2002;60: 881-886.

5. Secretaria de Assistência à Saúde, Ministério da Saúde. Portaria ${ }^{\circ} 97$. Diário Oficial da União 23 de março de 2001.

6. Poser CM, Paty DW, Scehinberg L, et al. New diagnostic criteria for multiple sclerosis: guidelines for research protocols. Ann Neurol 1983;36:227-231.

7. Kurtzke JF. Rating neurological impairment in multiple sclerosis: an expanded disability status scale (EDSS). Neurology 1983;33:1444-1452.

8. Polman $\mathrm{CH}$, Reingold SC, Edan G, et al. Diagnostic criteria for multiple sclerosis: 2005 revisions to the "McDonald Criteria" . Ann Neurol 2005;58:840-846.

9. Callegaro D. Contribuição ao estudo clínico evolutivo da esclerose múltipla: análise de 120 pacientes. Tese. Universidade de São Paulo. São Paulo, 1989.

10. Leite ACCB, Andrade C, Novis S. Esclerose múltipla no Rio de Janeiro: apresentação clínica em 51 casos. Arq Neuropsiquiatr 1990;48(Supl): S66.

11. Lana-Peixoto M, Lana-Peixoto MIV. Is multiple sclerosis in Brazil and Asia alike? Arq Neuropsiquiatr 1992;50:119-125.

12. Tilbery CP, Felipe E, Baldauf CM, Peres MFP. Esclerose múltipla: análise clínica e evolutiva de 214 casos. Arq Neuropsiquiatr 1995;53:203-207.

13. Papaiz-Alvarenga RM, Santos CMM, Abreu JS, et al. Esclerose múltipla: perfil clínico e evolutivo no município do Rio de Janeiro. Rev Bras Neurol 1995;31:75-87.

14. Oliveira EML, Annes M, Oliveira ASB, Gabbai AA. Esclerose múltipla: estudo clínico de 50 pacientes acompanhados no Ambulatório de Neurologia UNIFESP-EPM. Arq Neuropsiquiatr 1999;57:51-55.

15. Moreira MA, Felipe E, Mendes MF, Tilbery CP. Esclerose múltipla: estudo descritivo de suas formas clínicas em 302 casos. Arq Neuropsiquiatr 2000;58:460-466.

16. Arruda WO, Scola RH, Teive HAG, Werneck LC. Multiple sclerosis: report on 200 cases from Curitiba, Southern Brazil and comparison with other Brazilian series. Arq Neuropsiquiatr 2001;59:165-170.

17. Brito Ferreira ML, Machado MI, Vilela ML, et al. Epidemiology of 118 cases of multiple sclerosis after 15 years of follow-up on the reference center of Hospital da Restauração, Recife, Pernambuco, Brazil. Arq Neuropsiquiatr 2004;62:1027-1032.

18. Fragoso YD, Fiore APP. Description and characteristics of 81 patients attendind the Reference Center for Multiple Sclerosis of the coastal region of the State of São Paulo, Brazil. Arq Neuropsiquiatr 2005;63:738-741.

19. Cardoso E, Fukuda T, Pereira J, et al. Clinical and epidemiological profile of multiple sclerosis in a reference center in the State of Bahia, Brazil. Arq Neuropsiquiatr 2006;64:727-730.

20. Grzesiuk AK. Características clínicas e epidemiológicas de 20 pacientes portadores de esclerose múltipla acompanhados em Cuiabá - Mato Grosso. Arq.Neuropsiquiatr 2006;64:635-638.

21. Minguetti G. Magnetic resonance imaging in multiple sclerosis: analysis of 270 cases. Arq. Neuropsiquiatr 2001;59:563-569.

22. Puccioni-Sohler M, Lavrado FP, Bastos RRG, Brandão CO, Papaiz-Alvarenga R. Multiple sclerosis: clinical and laboratorial correlation. Arq Neuropsiquiatr 2001;59:89-91. 